\title{
Immediate endovascular treatment of blunt aortic injury: Our therapeutic strategy
}

Andrea Agostinelli, MD, ${ }^{\text {a }}$ Stefano Saccani, MD, ${ }^{a}$ Bruno Borrello, MD, ${ }^{a}$ Francesco Nicolini, MD, ${ }^{a}$ Pietro Larini, MD, and Tiziano Gherli, MD ${ }^{\mathrm{a}}$

From the Cardiac Surgery Department ${ }^{\mathrm{a}}$ and the Department of Radiology, ${ }^{\mathrm{b}}$ University of Parma, Parma, Italy.

Received for publication Sept 19, 2005; revisions received Nov 26, 2005; accepted for publication Dec 9, 2005.

Address for reprints: Dr Andrea Agostinelli, Cardiac Surgery Department, Ospedale Maggiore, Via Gramsci 14, 43100 Parma, Italy (E-mail: andrea.agostinelli@tiscali.it).

J Thorac Cardiovasc Surg 2006;131:1053-7

0022-5223/ $\$ 32.00$

Copyright $(\odot) 2006$ by The American Association for Thoracic Surgery

doi:10.1016/j.jtcvs.2005.12.015
Background: Posttraumatic aortic rupture is a potentially lethal injury. Endovascular procedure has recently proved to be a valid option. Timing of the treatment, however, is still a debated issue. We evaluated the feasibility and safety of immediate stent-graft repair of acute posttraumatic aortic injury.

Methods: From 1998 to 2005, 15 patients (11 men and 4 women, mean age 42.3 years) with blunt aortic injury were treated with immediate stent-graft positioning. In patients with clinical and radiologic signs of impending rupture, endovascular treatment was performed in an emergency setting (11 cases). In the 4 remaining patients the aortic lesion was treated after clinical management. When present, immediate life-threatening nonaortic lesions were treated before endovascular stenting ( 6 cases). In 1 case emergency laparotomy and endovascular procedure were performed simultaneously. Stent positioning was monitored by intraoperative transesophageal echocardiography in all cases.

Results: Endovascular procedure was successful in $100 \%$ of the patients. Two patients died perioperatively as a consequence of a multiorgan failure. Both patients were in American Society of Anesthetists class V and were in severe intractable hemorrhagic shock before the procedure. Computed tomography scan performed before discharge showed correct positioning of the stent graft and absence of endoleaks in all cases. At a mean follow-up of 29 months (range 1-79) all patients were alive but 1 , who died of unrelated cause, and no intervention-related complication had occurred.

Conclusions: Immediate stent-graft repair of posttraumatic aortic injury is a feasible and safe procedure. It allows us to minimize the surgical risks and to treat stable and unstable lesions even when associated lesions would contraindicate traditional surgical intervention.

B lunt aortic injury (BAI) is the second greatest cause of posttraumatic death, and it is often fatal in the early hours after injury. ${ }^{1,2}$ In the great majority of the cases it is caused by rapid deceleration in road accidents, and patients have severe multiple trauma such as craniocerebral trauma, thoracic trauma with lung contusion and multiple rib fractures, intra-abdominal parenchymatous bleeding lesions, and bone fractures. Despite recent advances in surgical and circulatory assistance techniques, early surgical repair, although often necessary, is still affected by high mortality and morbidity rates ${ }^{3,4}$ or is not feasible due to the presence of associated lesions.

Since the first report in 1994 by Dake and colleagues, ${ }^{5}$ endovascular stent grafting has emerged as a valid, less invasive alternative to open surgical treatment for thoracic aorta diseases. Some authors have recently demonstrated the safety and effectiveness of endovascular stent-graft repair of BAI, even in patients with severe polytrauma. ${ }^{6,7}$ However, optimal timing of the treatment and management of the 


\section{Abbreviations and Acronyms \\ $\mathrm{BAI}=$ blunt aortic injury \\ $\mathrm{CT}=$ computed tomography}

associated lesions is still debated. According to some authors, delayed treatment is associated with better results. ${ }^{8,9}$ The purpose of the current study was to prospectively evaluate the early and medium-term results of immediate endovascular repair of BAI in patients with severe polytrauma.

\section{Material and Methods}

From 1998 to December 2004, 15 patients had descending aorta transluminal stent-graft repair for acute BAI. In the same period, at our institution, a total of 97 thoracic endovascular procedures were performed. The patients' mean age was 42 years (range 22-69) and 11 patients were men. The cause of the injury was rapid deceleration from blunt trauma due to road accidents in 14 cases ( 8 car accidents, 5 motorcycle accidents, 1 bicycle accident) and to a fall from a tree in the remaining case.

All patients suffered from severe trauma with multiple associated lesions including: lung contusion and/or multiple rib fractures with pneumo- and/or hemothorax $(\mathrm{n}=13)$, blunt cerebral contusion $(n=6)$, liver laceration $(n=2)$, spleen injury $(n=3)$, atriocaval laceration $(n=1)$, spine fractures with $(n=2)$ or without $(n=1)$ spinal cord injury, pelvic fractures with major hematoma $(\mathrm{n}=2)$.

Hemodynamic instability and shock, defined as a systolic arterial pressure $<90 \mathrm{~mm} \mathrm{Hg}$ and cardiac pulse $>120$ beats/ min, was present in 10 patients and in 2 was not reversed after fluid management (infusion of $2000 \mathrm{~mL}$ crystalloids) and administration of vasoconstrictors (norepinephrine, epinephrine). One patient had cardiac tamponade; 5 were unconscious (Glasgow Coma Score $<8$ ).

Aortic disruption was detected at computed tomography (CT) scan in 14 cases. In 1 patient with cardiac tamponade who had emergency sternotomy for an aortocaval laceration, the rupture was diagnosed at intraoperative transesophageal echocardiography. In 2 patients, additional digital subtraction angiography was performed to confirm the diagnosis and assess supra-aortic vessel involvement.

\section{Therapeutic Protocol and Stent-Graft Procedure}

All patients were diagnosed in the acute phase. Stent-graft repair was considered as the first-choice treatment in all anatomically suitable patients with acute aortic disruption. Lack of straightness of the iliac-femoral axis was not considered a contraindication to stent positioning. Contraindications to endovascular treatment were proximal neck length less than $0.5 \mathrm{~cm}$ and transverse aortic diameter not suitable for available endoprosthesis. Following Advanced Trauma Life Support guidelines, any imminently lifethreatening injury such as abdominal or intracranial hemorrhage was treated before stent-graft implantation. Patients with imaging findings and/or clinical signs that suggested impending rupture had

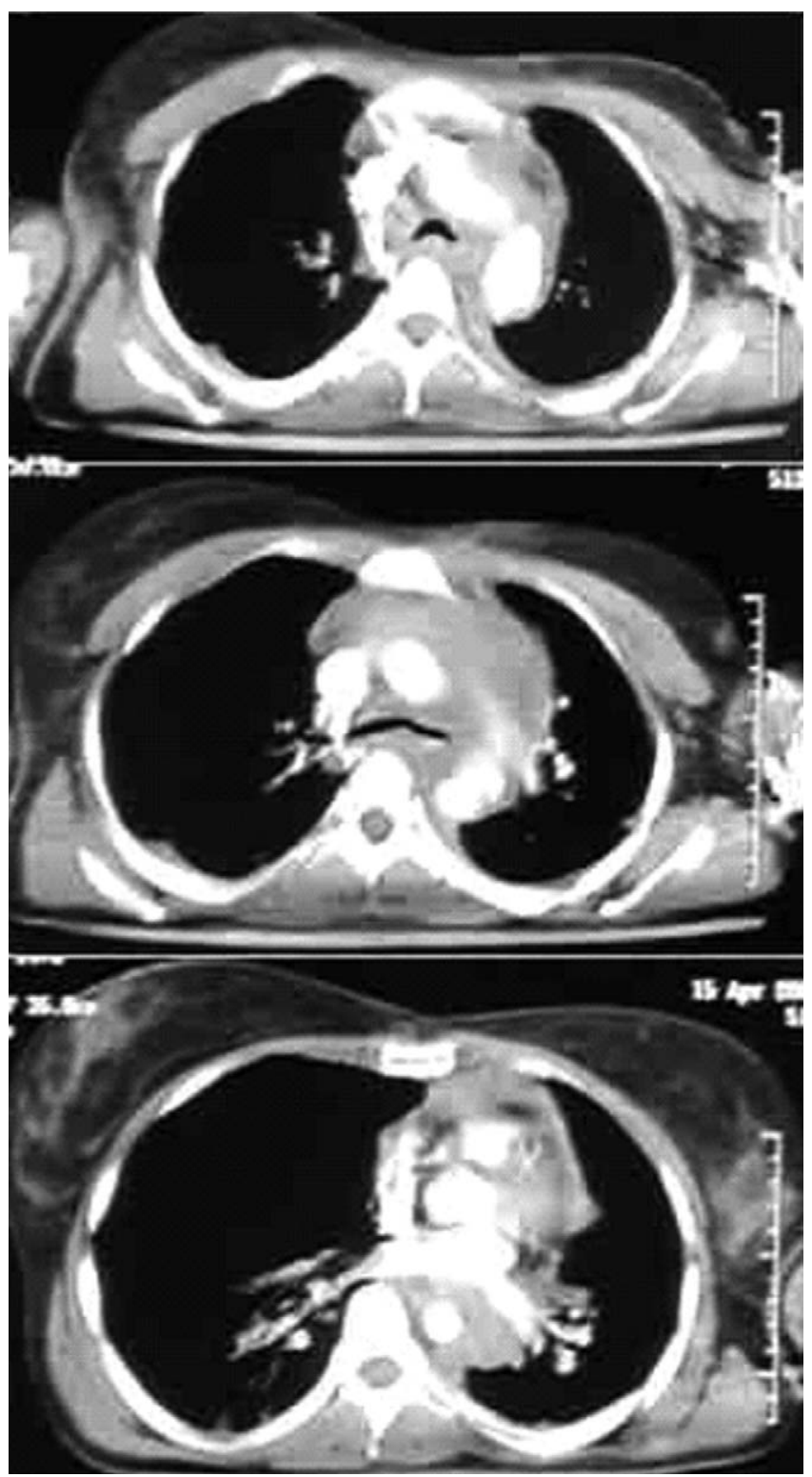

Figure 1. Preoperative CT scan showing posttraumatic disruption of aortic isthmus with large periaortic and mediastinal hematoma suggesting impending rupture.

emergency endovascular repair. The imaging findings included: periaortic and mediastinal hematoma, hemorrhagic pleural effusion, discontinuity of the aortic contour (Figure 1). Clinical signs included: pseudocoarctation syndrome, hemorrhagic shock with massive pleural effusion $(>1500 \mathrm{~mL})$. In patients with both imminently life-threatening lesions and unstable aortic disruption, a simultaneous surgical and endovascular approach was attempted when feasible. Stable aortic lesions were treated after fluid management, adequate clinical stabilization, and treatment of associate lesion.

Stent procedures were performed in a cardiac surgery operating theater by a combined team of cardiac surgeons and radiologists, 
with patients under general anesthesia. All procedures were monitored with a portable Philips BV $300 \mathrm{C}$-arm image intensifier with digital subtraction (Philips Medical System, Eindhoven, Netherlands) and by an echocardiograph (Vigment System Five, Horten, Norway) equipped with a multiplanar transesophageal probe. A pigtail catheter was introduced into the ascending aorta through a right brachial approach for pre- and postoperative aortography. Depending on the risk of bleeding, a maximum dose of 5000 UI of heparin was administered. In all patients a Talent self-expanding endograft (Medtronic AVE, Santa Rosa, Calif.) was implanted. Thirteen patients required 1 segment and 2 patients required 2 . The size of the prosthesis was determined on the basis of the CT scan images. The grafts were $15 \%$ to $20 \%$ larger than the adjacent normal aorta transverse diameter. In our series, the average prosthesis size was 28 (range 26 to 34 ). In 2 patients with short proximal neck, the left subclavian artery was covered with no postoperative complications. Vascular access was obtained by surgical exposure of the common femoral artery in 12 patients, of the external iliac artery in 2, and of the infrarenal aorta in 1. Systemic hypotension was induced during deployment of the stent graft to avoid graft displacement. After the procedure was completed, a digital subtraction angiography and echocardiography with color-flow mapping were performed to verify the correct positioning of the stent and to detect any primary endoleak. Follow-up consisted of CT scan before hospital discharge, at 1, 3 and 6 months, and yearly thereafter.

\section{Results}

All the 15 patients admitted to our institution from 1998 with diagnosis of BAI met the criteria for endovascular treatment, and none of them had traditional surgical repair. Following our protocol, 11 patients had emergency stentgraft repair of the aortic disruption due to the presence of radiologic or clinical signs suggesting impending rupture. In 5 of these patients stent grafting was performed after emergency surgical treatment of coexisting nonaortic lifethreatening lesions: splenectomy in 3 patients, liver suture in 1 , and simultaneous liver suture and aortocaval laceration suture through median sternotomy in the remaining patient, who had abdominal hemorrhage and cardiac tamponade. One patient with hemodynamic instability for abdominal hemorrhage and signs of impending rupture of thoracic aorta had simultaneous laparotomy and stent grafting through abdominal aorta. The same patient also had a brachiocephalic artery pseudoaneurysm involving the origin of the right carotid artery; a few days after stent-graft implantation the patient had a right carotid to right subclavian artery surgical translocation and the apposition of 2 partially overlapping balloon expandable stent grafts (Jomed) in the innominate artery. The left common carotid artery, contiguous to the origin of the brachiocephalic trunk, was protected from possible superimposition by a bare stent (Figure 2).

The remaining 4 patients were treated after clinical stabilization and did not require any additional surgery. The mean time from the diagnosis to treatment was 19.4 hours

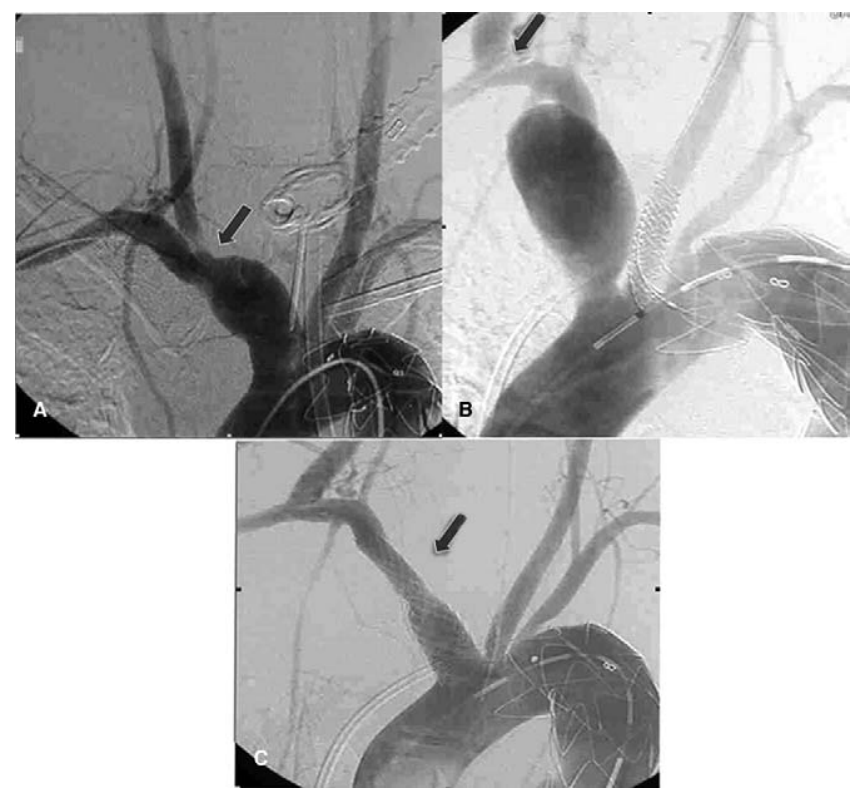

Figure 2. A, Intraoperative angiographic control after stent-graft deployment in the thoracic aorta showing innominate artery pseudoaneurysm and intimal flap in the right common carotid artery (black arrow). B, Angiographic control after surgical relocation of the right carotid artery on the right subclavian artery (black arrow). C, Angiographic control after stenting of the innominate artery (black arrow).

(range 3 to 122 hours). In all patients stent graft was placed without any difficulty and immediate technical success rate was $100 \%$, as the aortic lesion could be excluded in all cases as intended. Two patients (13\%) died perioperatively. Death occurred a few hours and 2 days, respectively, after the procedure as a consequence of a posttraumatic multiorgan failure. Both patients had multiple severe thoracoabdominal lesions and were in American Society of Anesthetists class $\mathrm{V}$; they were treated in the emergency room due to the presence of severe hemorrhagic shock that was not responsive to fluid management and vasoconstrictor administration. One patient also required an explorative laparotomy with liver suture before graft implantation. In both patients, endovascular procedure was carried out successfully.

Intensive care unit stay ranged from 1 to 30 days (mean stay 8 days) and was related to the severity of associated lesions. Ten patients $(67 \%)$ required prolonged ventilation, and acute reversible renal failure (serum creatinine $>1.5$ $\mathrm{mg} / \mathrm{dL})$ occurred in 4 patients $(27 \%)$. There were no neurologic complications (paraplegia or stroke) after the procedures. One patient had paraplegia before stent implantation due to thoracic spine myelic fracture. No other major complication, including embolization or stent migration, occurred. A postimplantation syndrome, consisting of leukocytosis and fever, was observed in all patients. 
Postoperative CT scans performed before discharge showed correct positioning of the stent-graft and complete sealing of aortic rupture in all 13 survivors. After a mean follow-up of 29 months (range 1 to 79) all patients are alive and in good health except for 1 who died of an unrelated cause (pulmonary cancer). At CT scan, no stent-graft failure, leak, or distal migration was detected in any of the 12 survivors.

\section{Discussion}

Timing of repair of BAI is still a debated issue. ${ }^{10-12}$ For several decades standard surgical practice has dictated that traumatic aortic rupture must be treated by immediate surgical repair. ${ }^{13}$ In fact, despite advances in surgical techniques, in severe polytrauma patients, immediate surgical repair of BAI is still affected by high mortality and morbidity rates and is often not even feasible. According to most recent reports, mortality ranges from $6.7 \%$ to $31 \%$ and paraplegia ranges from $0 \%$ to $36.4 \% .^{2-4,14,15}$ In the past few years some authors have suggested postponing surgical treatment until after medical stabilization of patients with multiple traumas to minimize mortality and morbidity rates connected with early repair. ${ }^{10,11,16-18}$

Since the first successful experience in the endovascular treatment of thoracic aorta, reported by Dake and colleagues in $1994,{ }^{5}$ endovascular technique has significantly improved and stent-graft repair has proved a safe and valid option for both acute and chronic pathologies. Recently many authors have reported success with early stent-graft repair of posttraumatic aortic lesions. ${ }^{19-22}$ Stent grafts allow surgeons to minimize or eliminate traumatism connected with open surgery. The patient is positioned supine; thoracotomy, onelung ventilation, cardiopulmonary bypass, and complete anticoagulation are not required. The thoracic aorta is not crossclamped and, because usually lesions involve short segments of thoracic aorta, only the proximal portion of the descending aorta needs to be covered, reducing the risks of postoperative paraplegia. Furthermore, patients with BAI are usually young: atherosclerotic disease of the aorto-iliacfemoral axis and of the proximal and distal landing zones is unlikely. Therefore, immediate treatment of BAI has become an attractive option, including for unstable patients with multiple traumas.

In the literature, however, there is no general agreement about the timing of treatment of BAI. Fattori and colleagues ${ }^{8}$ recently reported their experience with delayed endovascular treatment of BAI. Among their 19 patients, 8 were diagnosed in the chronic phase. Of the remaining 11 cases, 9 were scheduled for a delayed treatment and 2 required emergency treatment for imaging signs of impending rupture. The authors concluded that patients with unstable lesions should be treated as an emergency and other patients should have delayed treatment after stabilization of nonaortic lesions.

Bortone and colleagues ${ }^{23}$ compared patients who had immediate and delayed endovascular treatment for acute type B aortic dissection and posttraumatic aortic disruption. They found that in 1 patient suffering from BAI who was scheduled for a delayed treatment, "surgical removal of the pseudoaneurysm was still necessary because of further compression of the airway stem." They concluded that immediate treatment allows safe management of all patients with complete healing of the aortic wall and regression of the pseudoaneurysm.

In our series all patients had multiple traumas. Ten patients had hemodynamic instability and shock. Blunt cerebral contusion was present in 6 cases and a major bleeding lesion in 8 (spleen injury, atriocaval laceration, liver laceration, and pelvic fractures). Mortality and morbidity connected to emergency traditional surgical repair would likely have been prohibitive in most of the patients. But according to radiologic and clinical criteria, immediate repair was required for suspected impending rupture in 11 patients. Stent grafting was feasible in these unstable patients too and meant risks connected with delayed treatment could be avoided.

The mean age in our series was 42 years and all patients presented healthy aorto-iliac-femoral axis. In only 2 cases 2 segments were implanted, and in the remaining 13 cases, 1 segment was sufficient to exclude the rupture. The subclavian artery was partially covered in 2 cases without clinical consequences. Endovascular procedure was carried out successfully and the aortic wall completely sealed in all cases. Two patients died perioperatively, but death was unrelated to the procedure. These data confirm that BAI is an ideal condition for endovascular treatment: it usually affects young patients with healthy peripheral arteries and involves a small portion of proximal descending aorta.

Following the Advanced Trauma Life Support Manual, all imminently life-threatening lesions were treated before aortic stenting. Sometimes, in patients with coexisting lifethreatening nonaortic lesions and signs of impending rupture, it is difficult to define a therapeutic priority. In our series, procedures were performed in a cardiac surgery operating theatre with a surgical and radiologic team. This strategy allowed us to perform combined surgical and endovascular treatment in high-risk patients in which therapeutic priority was uncertain. One patient with bleeding liver lesion and signs of impending aortic rupture had simultaneous liver suture and aortic stent grafting. Another patient with cardiac tamponade had full sternotomy for atriocaval suture and, after an intraoperative transesophageal echocardiography revealed BAI, had immediate endovascular repair. Other patients with both major bleeding lesions and unstable aortic lesions had surgical repair of 
nonaortic lesions in less specialized hospitals before admittance to our trauma center.

At a mean follow-up of 29 months, no major complications, including perigraft leak, graft migration, or distal embolization, were encountered. This confirms the anatomical suitability of BAI for endovascular treatment. From our experience it appears that with early treatment, mortality is connected to severity of trauma and associated lesions rather than to the procedure itself.

The comparatively low number of patients constitutes a limitation of our study, and further follow-up is mandatory to confirm our results. However, we can conclude that due to its low degree of invasiveness and the anatomical suitability of the lesion, immediate endovascular stent graft of acute posttraumatic aortic rupture appears to be safe and effective. It is particularly suitable for patients with severe polytrauma, unstable aortic lesions, and major associated lesions, in which case it is probably the first-choice treatment. If procedures are carried out in a cardiac surgery operating theatre, combined endovascular and surgical treatment is feasible. It appears to be a valid option for patients with coexisting life-threatening nonaortic lesions and signs of impending aortic rupture, cases where it is hard to define the priority of treatment.

Endovascular treatment is a relatively new procedure; the first application in the thoracic aorta was described in $1994,{ }^{5}$ and recently some authors reported high rates of complication, albeit in particular types of patient. ${ }^{24,25}$ So further studies on larger number of patients, and with longer follow-up periods, are necessary to definitively validate this technique, especially applied to a young population of patients like ours. Further studies on patients with stable posttraumatic aortic lesions are also required to assess the optimal timing of treatment, whether early or delayed.

\section{References}

1. Smith RS, Chang FC. Traumatic rupture of the aorta: still a lethal injury. Am J Surg. 1986;152:660-3.

2. Fabian TC, Richardson JD, Croce MA, Smith JS Jr, Rodman G Jr, Kearney PA, et al. Prospective study of blunt injury: multicenter trial of the American Association for Surgery of Trauma. J Trauma. 1997; 42:374-80.

3. von Oppel UO, Dunne TT, De Groot MK P, Zilla P. Traumatic rupture of the aorta: twenty year matanalysis of mortality and risk of paraplegia. Ann Thorac Surg. 1994;58:585-93.

4. Attar S, Cardarelli MG, Downing SW, et al. Traumatic rupture of the aorta: recent outcome with regards to neurologic deficit. Ann Thorac Surg. 1999;67:959-65.

5. Dake MD, Miller DC, Semba CP, Mitchell RS, Walker PJ, Liddel RP. Transluminal placement of endovascular stent-grafts for the treatment of descending thoracic aortic aneurysms. $N$ Engl $J$ Med. 1994;331:1729-34.

6. Doss M, Balzer J, Martens S, et al. Surgical versus endovascular treatment of acute thoracic aortic rupture: a single-centre experience. Ann Thorac Surg. 2003;76:1465-70.

7. Lachat M, Pfammatter T, Witzke H, et al. Acute traumatic aortic rupture: early stent-graft repair. Eur J Cardiothorac Surg. 2002;21: 959-63.

8. Fattori R, Napoli G, Lovato L, et al. Indications for, timing of, and results of catheter-based treatment of traumatic injury to the aorta. AJR Am J Roentgenol. 2002;179:693-9.

9. Rosseau H, Soula P, Perrault P, et al. Delayed treatment of traumatic rupture of the thoracic aorta with endoluminal covered stent. Circulation. 1999;99:498-504.

10. Maggisano R, Nathens A, Alexandrova NA, et al. Traumatic rupture of the thoracic aorta: should one always operate immediately? Ann Vasc Surg. 1995;9:44-52.

11. Pate JW, Fabian TC, Walker W, et al. Traumatic rupture of the aortic isthmus: an emergency? World J Surg. 1995;19:119-26.

12. Akins CW, Buckley MJ, Dagget W, Mclduff JB, Austen WG. Acute traumatic aortic disruption of the thoracic aorta: a ten year experience. Ann Thorac Cardiovasc Surg. 1981;31:305-9.

13. Nagy K, Fabian T, Rodman G, Fulda G, Rodriguez A, Mirvis S. Guidelines for the diagnosis and management of blunt aortic injury: an EAST practice management guidelines work group. J Trauma. 2000; 48:1128-43.

14. Cowley RA, Turney SZ, Hankins JR, Rodriguez A, Attar S, Shanskar BS. Rupture of the thoracic aorta caused by blunt trauma. J Thorac Cardiovasc Surg. 1990;100:652-61.

15. Jamieson WRE, Janusz MT, Gudas VM, Burr LH, Fradet G, Henderson $\mathrm{C}$. Traumatic rupture of the thoracic aorta: third decade of experience. Am J Surg. 2002;183:571-5.

16. Fattori R, Celletti F, Bertaccini P, et al. Delayed surgery of traumatic aortic rupture: role of magnetic resonance imaging. Circulation. 1996; 94:2865-70

17. Galli R, Pacini D, Di Bartolomeo R, et al. Surgical indication and timing of repair of traumatic aortic ruptures of the aorta. Ann Thorac Surg. 1998;62:462-4.

18. Fattori R, Celletti F, Descovich B, et al. Evolution of post-traumatic aneurysm in the subacute phase: magnetic resonance imaging follow-up as a support of the surgical timing. Eur J Cardiothorac Surg. 1998; 13:582-7.

19. Orford VP, Atkinson NR, Thomson K, et al. Blunt traumatic aortic transaction: the endovascular experience. Ann Thorac Surg. 2003;75: 106-12.

20. Marty-Anè CH, Berthet JP, Branchereau P, Mary H, Arlic P. Endovascular repair of acute traumatic rupture of the thoracic aorta. Ann Thorac Surg. 2003;75:1802-7.

21. Iannelli G, Piscione F, Di tommaso L, Monaco M, Chiariello M, Spampinato N. Toracic aortic emergencies: impact of endovascular surgery. Ann Thorac Surg. 2004;77:591-6.

22. Morishita K, Kurimoto Y, Kawaharada N, et al. Descending thoracic aortic rupture: role of endovascular stent-grafting. Ann Thorac Surg. 2004;78:1630-4.

23. Bortone A, Schena S, D'Agostino D, et al. Immediate versus delayed endovascular treatment of post-traumatic pseudoaneurysm and type B dissections: retrospective analysis and premises to the upcoming European trial. Circulation. 2002;106(suppl I);I-234-40.

24. Makaroun MS, Dillavou ED, Kee ST, et al. Endovascular treatment of thoracic aortic aneurysms: results of phase II multicenter trial of the GORE TAG thoracic endoprosthesis. J Vasc Surg. 2005;41:1-9.

25. Cheung AT, Pochettino A, McGarvey ML, et al. Strategies to manage paraplegia risk after endovascular stent repair of descending thoracic aortic aneurysms. Ann Thorac Surg. 2005;80:1280-9. 